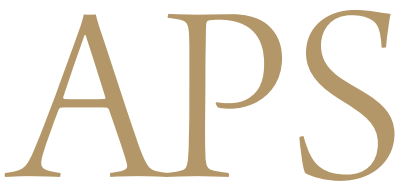

Archives of Plastic Surgery

\title{
A Pilot Study of Skin Resurfacing Using the 2,790-nm Erbium:YSGG Laser System
}

\author{
Jong Won Rhie ${ }^{1}$, Jeong Su Shim ${ }^{1}$, Won Seok Choi ${ }^{2}$ \\ ${ }^{1}$ Department of Plastic and Reconstructive Surgery, Daegu Catholic University Medical Center, Daegu; ${ }^{2}$ V Plastic Surgery, Daegu, Korea
}

Background The erbium:yttrium scandium gallium garnet (Er:YSGG) laser differs from other laser techniques by having a faster and higher cure rate. Since the Er:YSGG laser causes an appropriate proportion of ablation and coagulation, it has advantages over the conventional carbon dioxide $\left(\mathrm{CO}_{2}\right)$ laser and the erbium-doped yttrium aluminum garnet (Er:YAG) laser, including heating tendencies and explosive vaporization. This research was conducted to explore the effects and safety of the Er:YSGG laser.

Methods Twenty patients participated in the pilot study of a resurfacing system using a 2,790$\mathrm{nm}$ Er:YSGG laser. All patients received facial treatment by the 2,790-nm Er:YSGG laser system (Cutera) twice with a 4-week interval. Wrinkle reduction, reduction in pigment inhomogeneity, and improvement in tone and texture were measured.

Results Study subjects included 15 women and five men. Re-epithelization occurred in all subjects 3 to 4 days after treatment, and wrinkle reduction, reduction in pigment inhomogeneity, and improvement in tone and texture within 6 months of treatment.

Conclusions The 2,790-nm YSGG laser technique had fewer complications and was effective in the improvement of scars, pores, wrinkles, and skin tone and color with one or two treatments. We expect this method to be effective for people with acne scars, pore scars, deep wrinkles, and uneven skin texture and color.

Keywords Laser therapy / Rejuvenation / Ablation techniques

Received: 7 Dec 2013 • Revised: 7 Apr 2014 • Accepted: 8 Apr 2014

pISSN: 2234-6163 • elSSN: 2234-6171 • http://dx.doi.org/10.5999/aps.2015.42.1.52 • Arch Plast Surg 2015;42:52-58
Correspondence: Jeong Su Shim Department of Plastic and Reconstructive Surgery, Daegu Catholic University Medical Center, Catholic University of Daegu School of Medicine, Duryugongwon-ro 17-gil, Nam-gu, Daegu 705-718, Korea Tel: +82-53-650-4578

Fax: +82-53-650-4584

E-mail: 21csue@hanmail.net

\section{INTRODUCTION}

The human desire for a young and attractive appearance is the driving force for the development of cosmetic surgery. Laser technology is widely used for cosmetic surgery, a result of the widespread use of the carbon dioxide $\left(\mathrm{CO}_{2}\right)$ laser resurfacing technique starting in the 1990s. Laser resurfacing creates a limited thermal injury at the dermis layer, leading to a healing process with new collagen formation [1].
$\mathrm{CO}_{2}$ laser resurfacing reduces wrinkles by producing a dermal injury (width, $20-80 \mu \mathrm{m}$ ) at the dermis layer. This technique has a long recovery time (10-14 days), which can increase if infection or hyperpigmentation follows [2,3]. Adverse side effects include infection, pigment alterations, long-lasting erythema, and scarring [4]. The high risk of significant treatment complications, such as infection, changes in pigmentation, scarring, and prolonged erythema associated with this technique has warranted the development of new treatment modalities capa- 
ble of providing safer and more consistent alternatives [5]. In 1997, the erbium-doped yttrium aluminum garnet (Er:YAG) laser was approved by the Food and Drug Administration, presenting an alternative to the $\mathrm{CO}_{2}$ laser, with a shorter recovery time and fewer complications [6]. This laser also has its problems. Since it creates only a $20-\mu \mathrm{m}$ or smaller dermal injury, it produces superficial ablation. This is less beneficial, and patients have discomfort such as oozing $[7,8]$. Therefore, a new laser resurfacing tool was needed.

The erbium:yttrium scandium gallium garnet (Er:YSGG) laser is a technique overcoming the shortcomings of other lasers with better results and shorter recovery time. The water absorption coefficient of this laser has a wavelength of $2,790 \mathrm{~nm}$, which is between that of the Er:YAG laser $(2,940 \mathrm{~nm})$ and that of the $\mathrm{CO}_{2}$ laser (10,600 nm) (Fig. 1) [9]. This means that the Er:YSGG laser has an ideal wavelength, balancing the vaporization of the epidermis layer and the residual heating of the dermis layer. Thus, the Er:YSGG laser has the advantages of both the $\mathrm{CO}_{2}$ laser and the Er:YAG laser. This study investigated the efficacy and safety of the Er:YSGG laser in a pilot study.

\section{METHODS}

\section{Patients}

The study subjects included 15 women and 5 men in the age range of 22 to 60 years. Each treatment was done after receiving informed consent from the patient.

\section{Laser treatment}

$5 \%$ lidocaine ointment was applied to the entire facial area at least 30 minutes before treatment. After wiping off the lidocaine with gauze, acetone was used to completely wipe it off. Laser therapy was done from the hairline to the chin. All patients received facial treatment by the 2,790-nm Er:YSGG laser system (Cutera, Brisbane, CA, USA) using parameters ranging from 1.5 to $3.5 \mathrm{~J} / \mathrm{cm}^{2}$ and a $400-\mu$ s pulse duration. Two sessions of treatment were performed with a 4-week interval. Pulses were applied using scan patterns consisting of 6-mm-diameter individual laser pulses with an overlap of $20 \%$ to avoid leaving any areas untreated.

\section{Photographic documentation}

Photographic documentation was performed at baseline and in the 20 patients who completed the two sessions at the pre-treatment, 1 day after the first treatment, 1 week after the first treatment, and 6 months after the final treatment. Standard lightening and views were used with a Nikon D80 SLR digital camera with a flash unit installed within the camera. All images were taken by the same photographer.

\section{Methods for data evaluation and collection}

Wrinkle reduction, reduction in pigment inhomogeneity, and improvement in tone and texture were measured. In the data measurement, pictures taken before the first procedure and those taken 6 months after the final procedure were compared. Evaluation of the pictures compared the pre-treatment and posttreatment pictures and assigned a numerical score (1-100). Three plastic surgeons who had no former information on the patients made the comparisons and assigned scores. The patients revisited the clinic and had pictures taken before the procedure, 1 day, 1 week, 1 month, and 6 months after treatment. Ay 6 months after the final procedure, the patients were asked to rate their satisfaction on a 4-point scale (very satisfied, satisfied, slightly satisfied, or unsatisfied). The collected data were statisti-

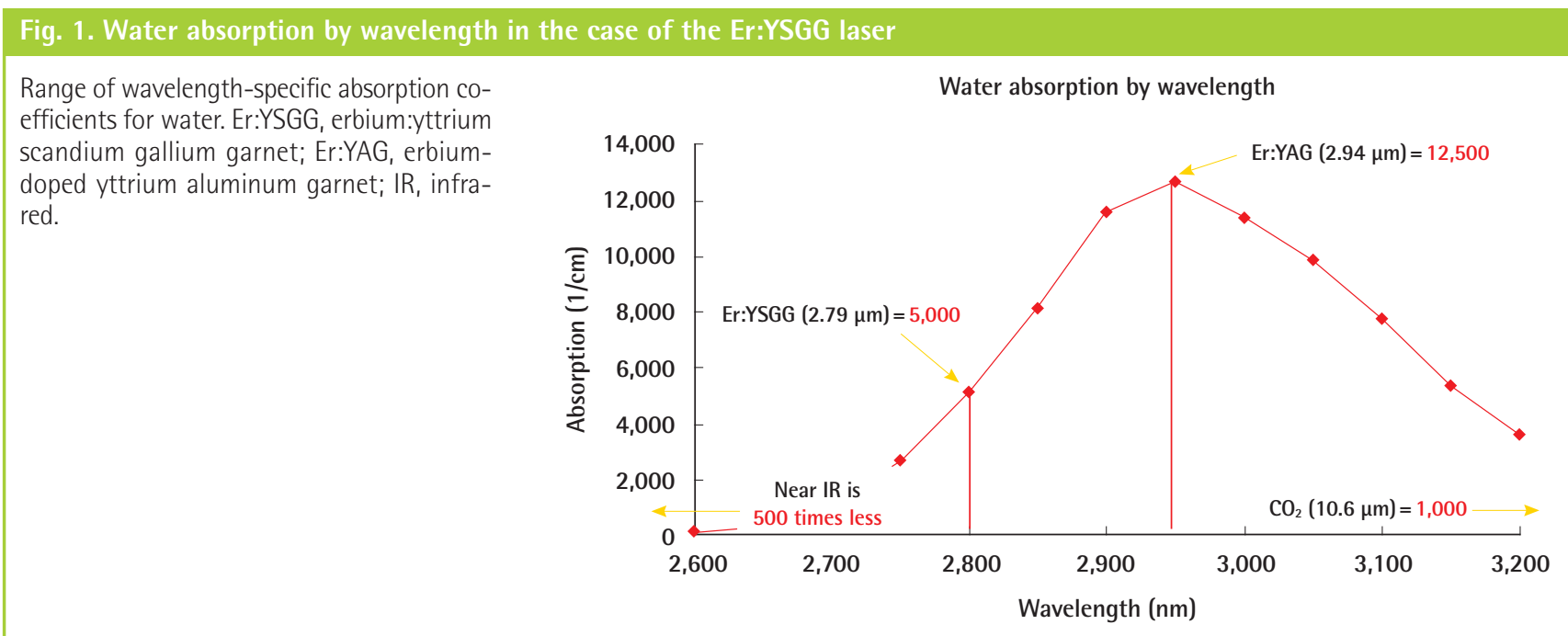


cally measured by using the mean value and the standard deviation (SD). Statistical significance testing used a one-sample ttest and a one-way analysis of variance, using SPSS ver. 19 (IBM Co., Armonk, NY, USA).

\section{Moisture, oil, and elasticity measurement}

Oil, moisture, and elasticity (Triple Sense, Moritex Co, Saitama, Japan) were measured before the procedure and 6 months after the final procedure was performed (Fig. 2). Evaluation compared the pre-treatment and post-treatment skin condition. In the dressing room, constant temperature and humidity were maintained for an accurate measurement of the skin condition. After cleansing the face, oil, moisture, and elasticity were measured. In order to reduce the measurement error, the measurements were taken twice on both of the patients' cheeks, and the average value was calculated. The collected data were statistically evaluated by using the mean value and the SD. Statistical significance testing used a paired-samples t-test, using SPSS ver. 19 (IBM Co.).

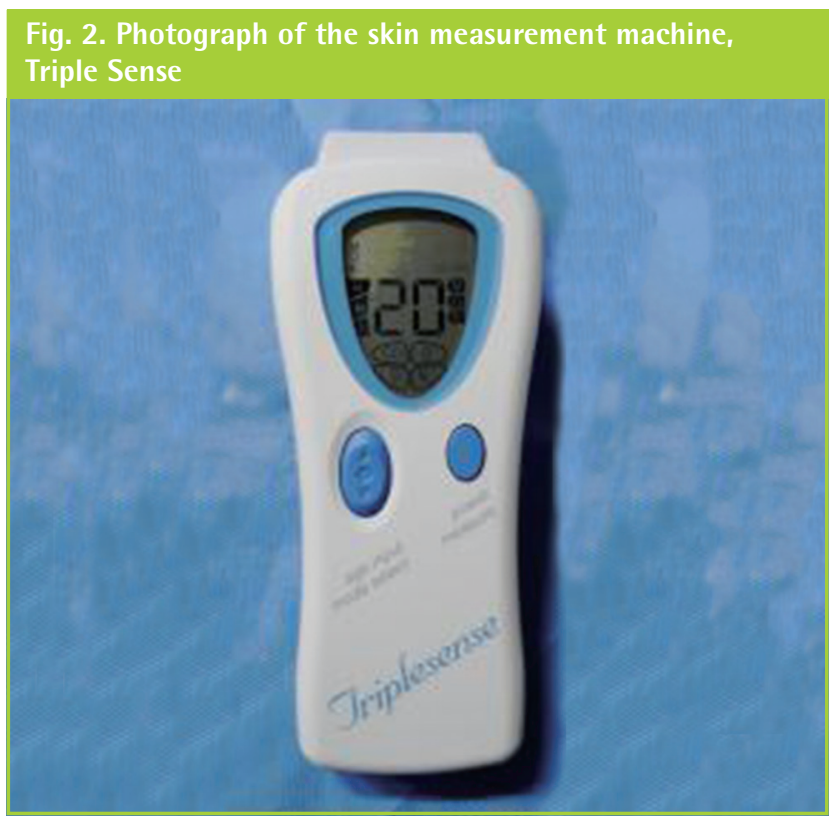

Table 1. Clinical scoring 6 months after the final Er:YSGG treatment

\begin{tabular}{|c|c|c|c|}
\hline Variable & $\mathrm{n}$ & Mean (standard deviation ) & P-value \\
\hline Wrinkle reduction & 60 & $18.617(3.325)$ & $0.000^{\mathrm{a})}$ \\
\hline $\begin{array}{l}\text { Reduction in pigment } \\
\text { inhomogeneities }\end{array}$ & 60 & $31.733(5.492)$ & 0.000 \\
\hline $\begin{array}{l}\text { Improvement in tone } \\
\text { and texture }\end{array}$ & 60 & $24.267(4.909)$ & 0.000 \\
\hline \multicolumn{4}{|c|}{$\begin{array}{l}\text { Evaluation by three plastic surgeons showed statistically significant changes in } \\
\text { wrinkle reduction, pigment inhomogeneity, and tone and texture improvement } \\
(P<0.05) \text {. } \\
\text { Er:YSGG, erbium:yttrium scandium gallium garnet. } \\
\text { a)Statistically significant with } P<0.05 \text {. }\end{array}$} \\
\hline
\end{tabular}

\section{RESULTS}

The study subjects included 15 women and 5 men, all of whom successfully completed the study. Re-epithelization occurred in all of the subjects over 3 to 4 days of treatment, and wrinkle reduction, reduction in pigment inhomogeneity, and improvement in tone and texture within 6 months of treatment. Subjects were examined 1 day, 1 week, 1 month, and 6 months after treatment. Discomfort disappeared for all subjects within 2 days after treatment, and all returned to work within a week.

All patients experienced temporary mild to moderate erythema that improved with time. One of the patients had contact dermatitis at the treatment site, which resolved after 3 days of topical corticosteroid therapy. Other common complications of laser therapy, including hypopigmentation, crusting, infection, long-standing erythema, scarring, and blistering did not occur.

The evaluations performed by three plastic surgeons differed significantly post-procedure in the case of wrinkle reduction, pigment inhomogeneity reduction, and tone and texture improvement $(\mathrm{P}<0.05)$ (Table 1) (Fig. 3). Reduction in pigment inhomogeneity was the most significant improvement, followed by improvement in tone and texture and that in wrinkle reduction. There were no significant differences by evaluator; thus, the physicians were objective in score assignment (Table 2). The evaluations showed statistically significant changes in moisture, oil, and elasticity improvement $(\mathrm{P}<0.05)$ (Table 3). Elasticity showed the most extensive improvement, followed by moisture and oil. As shown in Table 4, the patients were satisfied in principle. This result agrees with that of the treatment effect evaluation by the physician-evaluators (Table 4).

Fig. 3. Clinical scoring 6 months after the final Er:YSGG treatment

Er:YSGG, erbium:yttrium scandium gallium garnet.

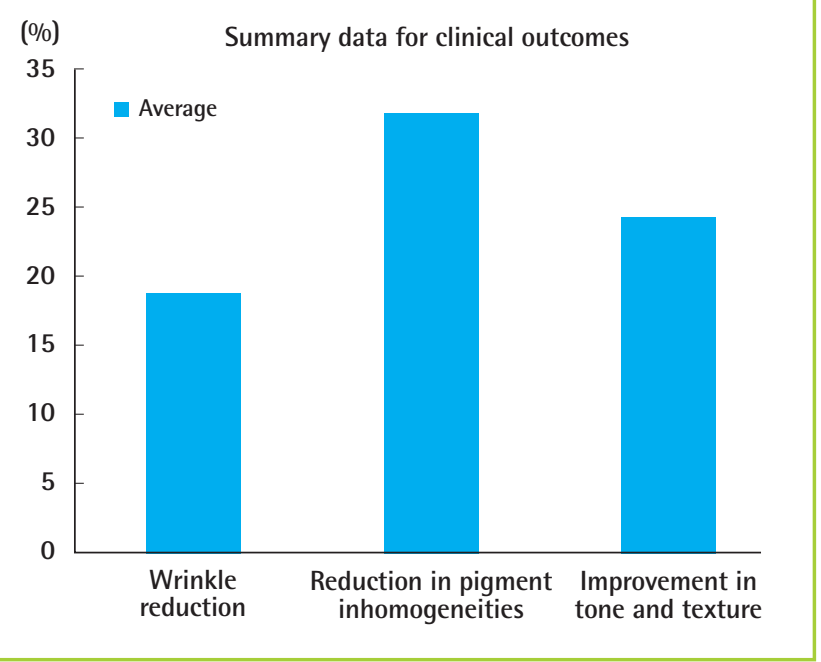


Table 2. Comparison of clinical scoring at 6 months by three plastic surgeons

\begin{tabular}{lcccc}
\hline \multirow{2}{*}{ Variable } & \multicolumn{3}{c}{ Plastic surgeons } & P-value \\
\cline { 2 - 4 } & $\mathbf{1}(\mathbf{n = 2 0 )}$ & $\mathbf{2}(\mathbf{n = 2 0 )}$ & $\mathbf{3} \mathbf{2 0})$ & $0.071^{\text {a) }}$ \\
\hline Wrinkle reduction & $18.6(3.284)$ & $17.5(3.236)$ & $19.9(3.167)$ & 0.099 \\
Reduction in pigment inhomogeneities & $32.2(5.976)$ & $29.7(4.703)$ & $33.4(5.344)$ & 0.127 \\
Improvement in tone and texture & $25.1(4.876)$ & $22.5(4.740)$ & $25.3(4.833)$ & \\
\hline
\end{tabular}

Values are presented as mean \pm standard deviation.

Scores did not differ significantly by the scoring physician (wrinkle reduction, reduction in pigment inhomogeneity, and improvement in tone and texture). Thus, the physicians were objective in their evaluation.

a)Statistically significant with $\mathrm{P}<0.05$.

Table 3. Objective scoring 6 months after final Er:YSGG treatment

\begin{tabular}{|lccc|}
\hline Variable & $\mathbf{n}$ & Mean (standard deviation ) & P-value \\
\hline Moisture difference & 10 & $8.8(2.251)$ & $0.000^{\mathrm{a})}$ \\
Oil difference & 10 & $-4.1(2.079)$ & 0.000 \\
Elasticity difference & 10 & $11.2(2.658)$ & 0.000 \\
\hline
\end{tabular}

Evaluation showed statistically significant changes in moisture, oil and elasticity improvement $(P<0.05)$. Only ten patients treated Er:YSGG were conducted. Er:YSGG, erbium:yttrium scandium gallium garnet.

a) Statistically significant with $\mathrm{P}<0.05$.

\section{Case 1}

A 28-year-old female received facial treatment by the 2,790-nm Er:YSGG laser system twice with a 4-week interval. Re-epithelization occurred in 1 week of treatment. The whole face showed greater improvement in tone and texture within 6 months of treatment. Complications of laser therapy did not occur. In the subjective self-assessment score, the patient was very satisfied (Fig. 4).

\section{Case 2}

A 31-year-old female received facial treatment by the 2,790-nm Er:YSGG laser system twice with a 4-week interval. Re-epithelization occurred in 1 week of treatment. In addition, natural healthy skin tone and texture was restored by 1 week of treatment. The whole face showed greater reduction in pigment inhomogeneity within 6 months of treatment. Complications of laser therapy did not occur. The subjective self-assessment score was satisfied (Fig. 5).

\section{DISCUSSION}

Laser therapy developed in response to a desire to reduce aging symptoms, including wrinkles, quickly and safely. The $\mathrm{CO}_{2}$ laser and Er:YAG laser have been common laser treatment techniques for years for treating wrinkles and improving the skin's condition. The wavelength of the Er:YAG laser is 2,940 nm, the closest wavelength to that of the water of the target chromo-
Table 4. Clinical scoring 6 months after the final Er:YSGG treatment

\begin{tabular}{|ccll|}
\hline No. & Sex/Age (yr) & $\begin{array}{c}\text { Patient } \\
\text { self-assessment }\end{array}$ & Adverse effect \\
\hline 1 & Female/28 & Very satisfied & None \\
2 & Female/31 & Satisfied & None \\
3 & Female/60 & Slightly satisfied & None \\
4 & Male/28 & Satisfied & None \\
5 & Female/30 & Unsatisfied & Contact dermatitis \\
6 & Male/22 & Very satisfied & None \\
7 & Female/34 & Satisfied & None \\
8 & Female/38 & Very satisfied & None \\
9 & Female/25 & Slightly satisfied & None \\
10 & Female/42 & Satisfied & None \\
11 & Female/58 & Satisfied & None \\
12 & Female/48 & Very satisfied & None \\
13 & Male/28 & Very satisfied & None \\
14 & Female/36 & Slightly satisfied & None \\
15 & Female/29 & Satisfied & None \\
16 & Male/33 & Very satisfied & None \\
17 & Female/43 & Satisfied & None \\
18 & Male/37 & Very satisfied & None \\
19 & Female/41 & Satisfied & None \\
20 & Female/55 & Satisfied & None \\
\hline Er:YSGG, erbium:yttrium scandium gallium garnet. & \\
\hline
\end{tabular}

phore $(3,000 \mathrm{~nm})$. Therefore, the Er:YAG causes mostly superficial ablation with little dermal effect. The $\mathrm{CO}_{2}$ laser delivers energy into the tissue, including the epidermal and the dermal layers. This results in better treatment with over-coagulation. Both of these techniques require a long recovery, with complications such as pain, infection, hyperpigmentation, long-standing erythema, and scarring. Patients want a more effective laser technique that has no side effects and a shorter recovery time.

The 2,790-nm YSGG laser overcomes the disadvantages of the older laser techniques. The Er:YSGG laser has an ideal wavelength, balancing the vaporization of the epidermis layer and the thermal damage to the superficial and upper layer of the dermis layer. This technique eradicates the epidermis 10 to 30 $\mu \mathrm{m}$ deep to control the vaporization and coagulation of the epidermis layer by delivering sufficient heat. The coagulated layer 
Fig. 4. Case 1 of the Er:YSGG laser treatment

A 28-year-old female received 2 treatments, 4 weeks apart. (A) Pretreatment. (B) One day after the first treatment. (C) One week after the first treatment. (D) Six months after the final treatment. Er:YSGG, erbium:yttrium scandium gallium garnet.
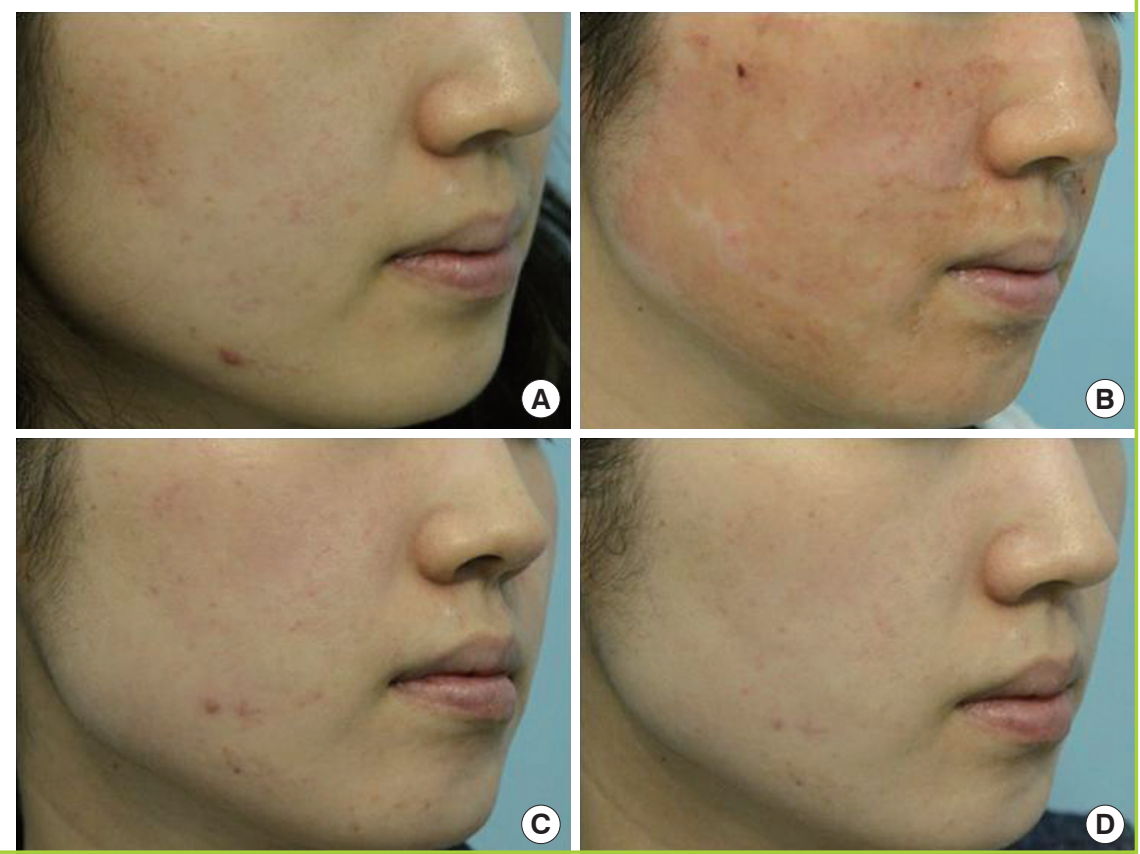

Fig. 5. Case 2 of the Er:YSGG laser treatment

A 31-year-old female received 2 treatments, 4 weeks apart. (A) Pretreatment. (B) One day after the first treatment. (C) One week after the first treatment. (D) Six months after the final treatment. Er:YSGG, erbium: yttrium scandium gallium garnet.
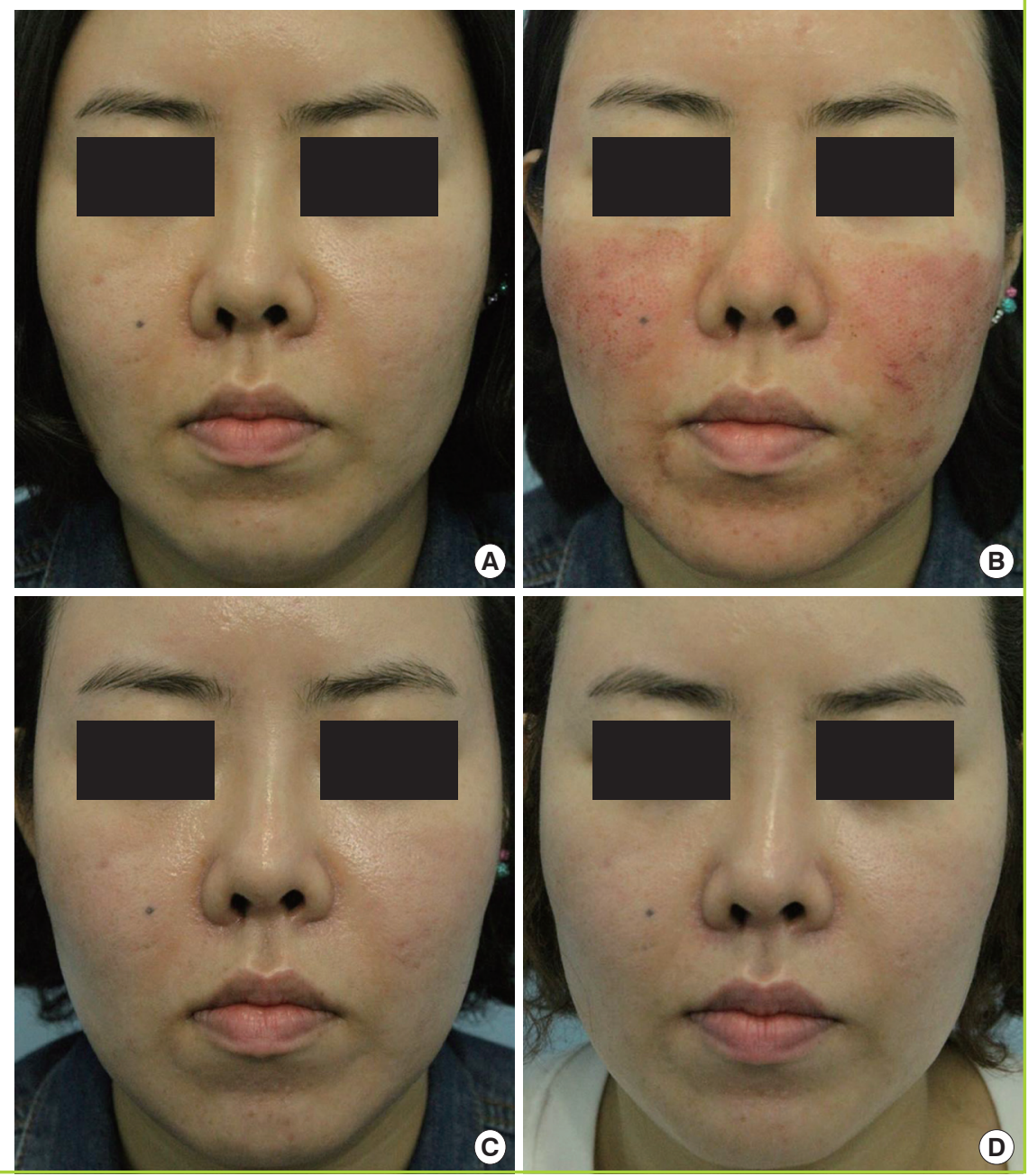
acts as a natural dressing and provides patient comfort and protection in the early healing period [8]. The thermal damage within the dermis layer stimulates the wound healing process with the end result of neocollagenesis and elastinogenesis in the proliferative stage of wound healing, followed by the remodeling process resulting in a tighter, better organized dermis, and removal of wrinkles in the epidermis. In this way, the 2,790-nm YSGG laser is able to treat wrinkles and improve the skin's condition (Table 5).

\section{Table 5. Comparison of thermal characteristics}

\begin{tabular}{lcll|}
\hline Characteristic & Er:YAG & Carbon dioxide & Er:YSGG \\
\hline Bleeding & YES & NO & NO \\
Pinpoint bleeding & YES & Occasional & Occasional \\
Coagulation & NO & Too much & YES \\
$\begin{array}{l}\text { Thermal stimulation } \\
\text { Excessive coagulation \& } \\
\text { risk of thermal overlap }\end{array}$ & NO & Too much & YES \\
Volume of tissue ablative & High & YES & NO \\
\hline
\end{tabular}

The ideal ratio between coagulation and ablation of the Er:YSGG laser results in the following: First, less bleeding. Second, expectation of collagen remodeling by coagulation promoted by thermal stimulation. Third, a lower risk of thermal overlap and complications. Fourth, better treatment results over a wider ablation area. Er:YAG, erbium-doped yttrium aluminum garnet; Er:YSGG, erbium:yttrium scandium gallium garnet.
The 2,790-nm YSGG laser is capable of two modes in the same wavelength: pearl laser and pearl fractional laser. Pearl laser improves the photodamage and texture of the skin by superficial resurfacing similar to the full epidermis renewal effect. The pearl fractional laser is effective in deep dermal ablation and creates a precise thermal wound while sparing the surrounding tissue. The wound healing response differs from that of ablative laser skin resurfacing because this method enables sparing of the epidermal tissue between thermal zones. The pearl fractional laser is capable of rapid re-epithelization. The combination therapy of pearl laser and pearl fractional laser can be synergistic. Combination therapy done once or twice has the same effect as 3 to 5 treatments with fractional laser therapy (Fig. 6) [10].

This study has its limitations as a pilot study, because it has a small test group and an insufficiently objective evaluation. In addition, it does not directly compare the 2,790-nm YSGG laser technique with the other two techniques, but the 2,790-nm YSGG laser has a shorter recovery time and fewer complications than the other ablative resurfacing techniques, with only one case of contact dermatitis. Furthermore, it offers better cosmetic results. This study showed successful results with only two treatments. The advantages of this technique are fewer treatments needed for obtaining the desired results and shorter

\section{Fig. 6. Er:YSGG pearl and pearl fractional laser treatment}

(A) A pearl fractional laser is effective in deep dermal ablation. (B) A pearl laser reduces photodamage and improves skin texture by superficial resurfacing (similar to the full epidermis renewal effect). Combination treatment with the pearl laser and pearl fractional laser is synergistic. Er:YSGG, erbium:yttrium scandium gallium garnet.
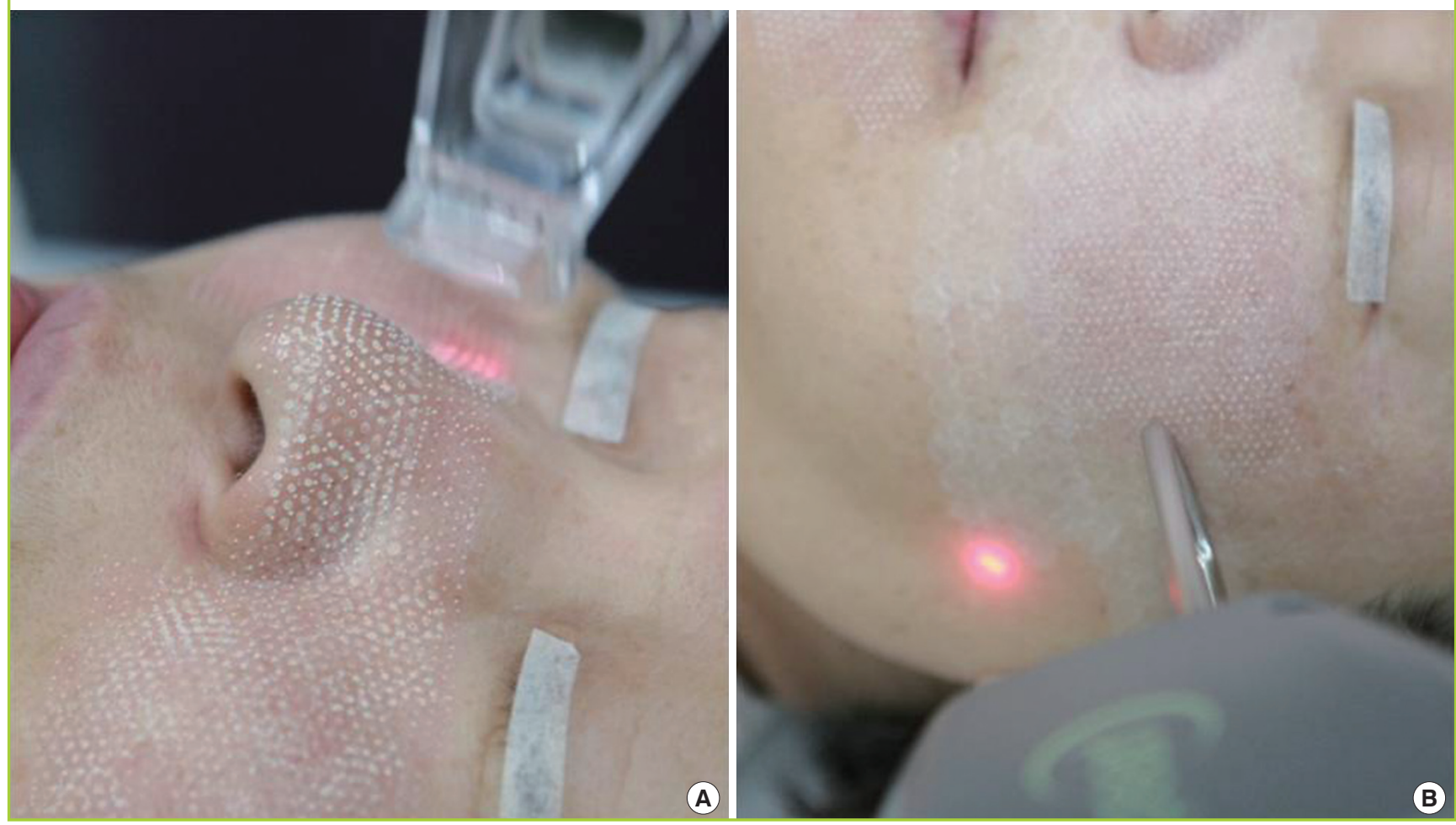
recovery time.

The 2,790-nm YSGG laser technique had fewer complications and was effective in scar treatment, pore treatment, wrinkle treatment, and skin texture and tone improvement within two treatments [11]. We expect this method to be effective for people with acne scars, pore scars, deep wrinkles, and uneven skin texture and color. Although additional controlled trials are needed for confirmation, the 2,790-nm YSGG laser is a promising laser technique for skin rejuvenation.

\section{REFERENCES}

1. Tay YK, Khoo BP, Tan E, et al. Long pulsed dye laser treatment of facial wrinkles. J Cosmet Laser Ther 2004;6:131-5.

2. Ross EV, Swann M, Soon S, et al. Full-face treatments with the 2790-nm erbium:YSGG laser system. J Drugs Dermatol 2009;8:248-52.

3. Marcells GN, Ellis DA. Laser facial skin resurfacing: discussion on erbium:YAG and CO2 lasers. J Otolaryngol 2000; 29:78-82.

4. Horton S, Alster TS. Preoperative and postoperative considerations for carbon dioxide laser resurfacing. Cutis 1999;64:
399-406.

5. Walgrave SE, Ortiz AE, MacFalls HT, et al. Evaluation of a novel fractional resurfacing device for treatment of acne scarring. Lasers Surg Med 2009;41:122-7.

6. Khatri KA, Gordon J, Garamela L. Laser skin resurfacing with a novel portable erbium:YAG laser. J Cosmet Laser Ther 2006;8:23-5.

7. Walsh JT Jr, Flotte TJ, Deutsch TF. Er:YAG laser ablation of tissue: effect of pulse duration and tissue type on thermal damage. Lasers Surg Med 1989;9:314-26.

8. Jaffe BH, Walsh JT Jr. Water flux from partial-thickness skin wounds: comparative study of the effects of Er:YAG and Ho:YAG lasers. Lasers Surg Med 1996;18:1-9.

9. Kaufmann R, Hartmann A, Hibst R. Cutting and skin-ablative properties of pulsed mid-infrared laser surgery. J Dermatol Surg Oncol 1994;20:112-8.

10. Tanzi EL, Wanitphakdeedecha R, Alster TS. Fraxel laser indications and long-term follow-up. Aesthet Surg J 2008;28: 675-8.

11. Dierickx CC, Khatri KA, Tannous ZS, et al. Micro-fractional ablative skin resurfacing with two novel erbium laser systems. Lasers Surg Med 2008;40:113-23. 